\title{
Electromyographic evaluation of the effect of ultrasound with muscle stretching in temporomandibular disorder: a clinical trial
}

\author{
Jalusa Boufleur ${ }^{1}$, Eliane Castilhos Rodrigues Corrêa ${ }^{1}$, Lais Chiodelli ${ }^{1}$, \\ Ana Maria Toniolo da Silva ${ }^{2}$, Lilian Gerdi Kittel Ries ${ }^{3}$
}

1Universidade Federal de Santa Maria - UFSM, School of Physical Therapy, Department of Physical Therapy and Rehabilitation, Santa Maria, RS, Brazil
'Universidade Federal de Santa Maria - UFSM, School of Speech Therapy, Department of Speech Therapy, Santa Maria, RS, Brazil
${ }^{3}$ Universidade do Estado de Santa Catarina - UDESC, School of Physical Therapy, Department of Physical Therapy, Florianópolis, SC, Brazil

\begin{abstract}
Aim: To investigate the electromyographic activity of masticatory muscles in women with myogenic or mixed TMD treated with ultrasound and ultrasound associated with stretching. Methods: Sixteen women with myogenic or mixed TMD, according to the Research Diagnostic Criteria for Temporomandibular Disorder (RDC/TMD), participated in the study. The patients were evaluated using surface electromyography (SEMG) of the masseter and anterior temporalis muscles, during maximum intercuspation, before and immediately after application of therapeutic resources. All patients were treated by ultrasound (US), ultrasound associated with stretching and placebo ultrasound, by turns, once a week with a one-week minimum interval between them. Results: There was greater symmetry of the masseter muscle electrical activity after ultrasound associated with stretching $(p=0.03)$. The electromyographic values for the maximum intercuspation as well as the symmetry of anterior temporal muscle $(p=0.47, p=0.84, p=0.84)$ and anteroposterior coefficient $(p=0.07, p=0.84, p=0.57)$ showed no statistically significant difference after the intervention, placebo, ultrasound and ultrasound with stretching. Conclusions: These results indicate that a single application of ultrasound and ultrasound associated with stretching were not able to modify the activity pattern of the masticatory muscles during maximum intercuspation, except the symmetry of the masseter muscle that increased with the last, in women with myogenic or mixed temporomandibular disorder.
\end{abstract}

Keywords: temporomandibular joint disorder; electromyography; ultrasonics; muscle stretching exercise.

\section{Introduction}

Received for publication: April 17, 2014 Accepted: June 11, 2014

Correspondence to: Jalusa Boufleur

Rua Machado de Assis, 221 apto. 402 CEP: $97050-450$ - Bairro Nossa Senhora das Dores Santa Maria-RS

Phone: + 5555 33476364; + 555591292052

E-mail: jalusaboufleur@yahoo.com.br
Temporomandibular disorder (TMD) is a multifactorial condition characterized by symptoms, most of them painful, which affect the stomatognathic system, including the ligaments, muscles and the temporomandibular joint $t^{1-2}$.

In the TMD treatment, non-invasive therapies such as physical therapy are recommend, which aims at relief the of musculoskeletal pain, improvement of inflammation and restoration of oral function ${ }^{3-4}$.

Ultrasound is one the physical therapeutic modalities used in the TMD treatment. It promotes pain relief and muscle relaxation, since muscle hyperactivity, 
considered one of the TMD etiologic factors, may lead to

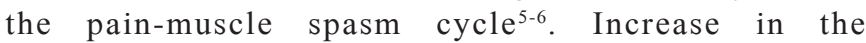
musculotendinous length and decrease of the muscle stiffness may be achieved by stretching 7 . Thus, the restoration of the musculotendinous length, associated to pain relief, may reflect on the masticatory muscle electromyographic (EMG) potentials during a certain activity, with the improvement of the muscular balance.

The muscle electrical activity at rest is higher in TMD patients, while the EMG potentials during the voluntary maximal contraction are reduced, compared to healthy subjects. Asymmetries also are observed in the masticatory muscles in these patients. The evaluation of the functional symmetry of the orofacial complex, involves the pattern of the mandibular movement and the masticatory muscles activity. The contraction pattern of a pair of muscles can be investigated by the surface $\mathrm{EMG}^{8-9}$.

Some authors ${ }^{10-11}$ have investigated the effect of specific physiotherapeutic protocols on the pain and on the functional limitation of TMD. Frequently, it is not known the isolated effect of a determined therapeutic modality on the signs and symptoms of the disorder and which of them was responsible for the patient's clinical improvement. The pain relief and mandibular range of movement increase obtained with the physical therapy, allows for a more effective and faster effect of the speech therapy and dental treatments on the functional restoration of the stomatognathic system ${ }^{12-13}$. Thereby, the aim of the present study was to evaluate the immediate effect of ultrasound, ultrasound combined with stretching of the mandibular elevator muscles and placebo on the activation pattern and symmetry of these muscles in women with myogenic and mixed TMD.

\section{Material and methods}

\section{Subjects}

The Clinical Trial Registration (NCT02139735) and the Research Ethics Committee of the Federal University of Santa Maria (protocol number 0281.0.243.000-08) approved the study and informed consent was obtained from each subject.

Twenty-nine prospective female subjects recruited from the UFSM Occlusal Clinical and from direct advertising for research subjects on electronic media were initially selected. Out of these, 16 women with mean age of $25.69 \pm 5.13$ years, with myogenic and mixed TMD took part in the experimental procedures of the research. All participants were treated by three therapeutic modalities (ultrasound, ultrasound with stretching and placebo). The modalities were applied in a random sequence (crossover design), with a one-week interval (washout period) among them, in order to dissipate the residual effect of the previous intervention ${ }^{14}$.

\section{Selection Criteria}

The inclusion criteria were: (1) age from 20 to 35 years old; (2) diagnosis of myogenic or mixed TMD, according the Research Diagnostic Criteria for Temporomandibular
Disorders (RDC/TMD). The exclusion criteria were: (1) neuropsicomotor disease; (2) history of trauma on the temporomandibular region, orofacial surgery or malformation; (3) consumption of anti-inflammatory, analgesic, antidepressant or myorelaxant drugs; (4) presence of acute pain in TMJ and (5) previous speech therapy or physical therapy (in the last six months). Additionally, to avoid the influence of the estrogenic hormone reduction ${ }^{15}$, the final period of the menstrual cycle (seven days before the menstruation) and the beginning of the menstruation (two first days) were skipped for the evaluation of women who did not use oral contraceptive and the ones who paused their use.

\section{Surface Electromyographic Signal Acquisition}

The electromyographic evaluation of the masticatory muscles (masseter and temporalis anterior, bilaterally) was conducted with the equipment Miotool 400 (Miotec ${ }^{\circledR}$; Porto Alegre, RS, Brasil), with four channels, 14-byte resolution, $2,000 \mathrm{~Hz}$ sampling frequency, $110 \mathrm{~dB}$ common mode rejection and a band pass filter of $20-500 \mathrm{~Hz}$. The EMG data were collected by Miograph $\left(\right.$ Miotec $^{\circledR}$ ) software and stored in a portable computer Acer, model Aspire 5250-0866.

During signal acquisition, the subjects were sat on a wooden chair, with their feet parallel on a rubber carpet, hands over the thighs and looking at a target placed at eye level in front of them.

Bipolar surface electrodes $\mathrm{Ag}-\mathrm{AgCl}, 20 \mathrm{~mm}$ center to center interelectrode distance (Hal Indústria Comércio de Metais Ltda,. São Paulo, SP, Brazil) connected to active sensors with differential input $\left(\right.$ Miotec $^{\circledR}$ ) were positioned on the region of greater volume of the muscle belly during maximum intercuspation position. Previous to the electrode placement, the skin was cleaned with gauze soaked with $70 \%$ alcohol (ISEK - International Society of Electromyography and Kinesiology). A common grounding to all channels was placed on the subject's sternum bone to avoid the interferences and noises in the EMG signal ${ }^{16}$.

The EMG recordings were acquired during maximum intercuspation (MI) and the patients were oriented to clench their teeth as much as possible and keep this contraction for 5 s. For the voluntary maximal contraction EMG recording, the patients were oriented in the same manner, but they clenched their teeth with Parafilm material, folded with similar width and thickness of a Trident chewing gum $(3.5 \times 1.5$ $\mathrm{cm})^{17}$, placed between the first and second molar, bilaterally. Each recording was repeated twice with a 2-min interval.

\section{Electromyographic Data Processing}

The EMG signal was processed in the time domain by the Root Mean Square (RMS). The best quality EMG signals were visually selected for posterior cutout and qualitative analysis. In all the recorded tests one second with the highest signal amplitude was selected from the total collection period.

The EMG signal normalization was performed using the maximal voluntary contraction as a reference value. Thus, the amplitude of the muscular activation of each muscle in 
the different situations was expressed as a percentage of the reference value, calculated using the following formula:

$$
x_{r m s}=\sqrt{\frac{1}{N} \sum_{i=1}^{N} x_{i}^{2}}
$$

where $x$ is the measurements data, $i$ is the measurements index and $N$ is the measurements length.

The percentage overlapping coefficient (POC\%) represents the pair of muscles (right and left side) symmetry, obtained by the overlapping of the normalized values of the amplitude EMG signal from the right and left sides. The anteroposterior coefficient (APC), which evaluates the balance between masseter and temporal muscles, was also calculated. This coefficient is the relation between the overlapping and non-overlapping areas obtained from the normalized values of the signal amplitude of the masseter and temporalis muscles from both sides. If only one pair of muscles contracts (for example: right and left masseter), there is an unbalanced contraction between these muscles, with an anterior or posterior displacement of the occlusal center, according the prevalence of the activity of the temporal or masseter muscles, respectively,16,18-20. It ranges from 0 (without balance) to $100 \%$ (complete balance).

For the POC and APC calculation, the EMG potentials were filtered with a band-pass of 20-500 Hz. The two coefficients were obtained with the mean potential calculated in RMS on the $25 \mathrm{~ms}$ moving windows (50 data). The data were processed with the Matlab (The MathWorks ${ }^{\circledR}$, R2006b version) software.

\section{Physical therapy procedures}

The subjects underwent three procedures: ultrasound, ultrasound with stretching and placebo. All of them were carried out with the patient lying supine with a towel roll under their cervical column.

Ultrasound: The Sonopulse III 1 and $3 \mathrm{MHz}$ model equipment (Ibramed Ltda., Amparo, SP, Brazil), set at $3 \mathrm{MHz}$, continuous mode and $1.0 \mathrm{~W} / \mathrm{cm}^{2}$ intensity, was applied during 6 $\min \left(3 \mathrm{~min}\right.$ on each side) at the TMJ and masseter muscle regions ${ }^{21}$.

Ultrasound simultaneous with masseter muscle stretching: The patient should open the mouth in a confortable position, keeping the lips closed, while the physical therapist applied a slight digital support on her mentum to keep the stretching during $30 \mathrm{~s}$ (adapted from Pertes and $\mathrm{Gross}^{21}(2005)$. The stretching was repeated twice with a 10 -s rest between the repetitions (Figure 1).

Ultrasound placebo: same procedures of the ultrasound group except that equipment was turned off.

\section{Statistical Analysis}

The effect of the three therapeutic interventions was evaluated in sixteen patients, comparing the results among them, before and after each intervention.

The STATISTICA 9.1 software was used for the statistical analysis. Data normality was verified by the Shapiro Wilk

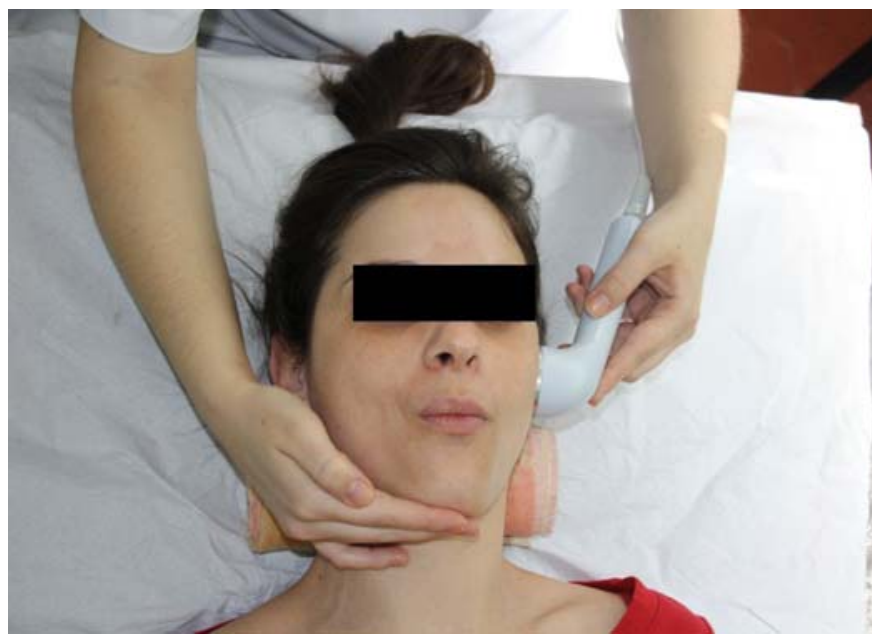

Fig. 1. Ultrasound with masseter muscle stretching

test. The comparison between the mean values of EMG signal amplitude before and after the intervention was carried out by the Student's t test for dependent samples or Wilcoxon test, according to the characteristic of the variables, parametric or non-parametric. Two-way ANOVA was used to compare the values of EMG amplitude before and after intervention, among the three modalities. A significance level of $5 \%$ was used.

\section{Results}

The comparative analysis of the EMG amplitude showed, for the three modalities, that there was no statistically significant difference between values obtained before and after intervention, during the maximum intercuspation, in all evaluated muscles (Table 1).

Table 1.Normalized values of EMG amplitude obtained during maximum intercuspation position, on the three modalities (ultrasound, ultrasound with stretching and placebo $(n=16)$

\begin{tabular}{lccc}
\hline Muscle & Pre & $\begin{array}{c}\text { Ultrasound } \\
\text { Post }\end{array}$ & p value \\
RM & $73.42 \pm 25.13$ & $74.38 \pm 29.30$ & 0.90 \\
LM & $72.88 \pm 31.13$ & $69.30 \pm 29.08$ & 0.52 \\
RAT & $90.59 \pm 29.12$ & $84.09 \pm 20.00$ & 0.20 \\
LAT & $85.70 \pm 36.46$ & $79.88 \pm 21.81$ & 0.39 \\
& \multicolumn{2}{c}{ Ultrasound with stretching } \\
Muscle & Pre & Post & p \\
RM & $74.21 \pm 28.23$ & $71.39 \pm 24.47$ & 0.41 \\
LM & $73.60 \pm 23.58$ & $72.11 \pm 25.86$ & 0.75 \\
RTA & $87.63 \pm 22.65$ & $84.53 \pm 20.99$ & 0.44 \\
LTA & $78.47 \pm 20.89$ & $83.21 \pm 24.79$ & 0.46 \\
& & Placebo & \\
Muscle & Pre & Post & p \\
MD & $68.16 \pm 21.56$ & $73.43 \pm 22.14$ & 0.19 \\
ME & $70.13 \pm 24.02$ & $74.17 \pm 22.03$ & 0.44 \\
RAT & $84.42 \pm 28.89$ & $87.54 \pm 23.19$ & 0.45 \\
LAT & $83.00 \pm 26.70$ & $83.72 \pm 23.54$ & 0.91 \\
\hline
\end{tabular}

Right masseter (RM), Left masseter (LE), Right anterior temporalis (RAT), Left anterior temporalis (LTA). Student's $t$ test for dependent samples. $p<0.05$ 
Table 2 - Differences of the normalized EMG potentials, during maximum intercuspation, before and after each intervention $(n=16)$

\begin{tabular}{lccc}
\hline Muscle & Treatment & $\begin{array}{c}\text { Difference pre and } \\
\text { post treatment }\end{array}$ & p value \\
Right Masseter & Ultrasound & 0.95 & \\
& Ultrasound+ stretching & -2.82 & 0.60 \\
& Placebo & 5.32 & \\
& Ultrasound & -3.58 & 0.51 \\
Left Masseter & Ultrasound+ stretching & -1.48 & \\
& Placebo & 4.04 & 0.26 \\
Right anterior temporalis & Ultrasound+ stretching & -3.10 & \\
& Placebo & 3.12 & \\
& & & \\
& & & \\
& Ultrasound & -5.82 & \\
Left anterior temporalis & Ultrasound+ stretching & 4.74 & \\
& Placebo & 0.71 &
\end{tabular}

Table 2 shows the differences pre- and post-intervention on the EMG potentials obtained among the modalities during the maximum intercuspation position for all the evaluated muscles.

Regarding the symmetry evaluation (POC) of the EMG amplitude, it was verified a statistically significant increase in the right and left masseter muscles with ultrasound and stretching. APC showed no difference after intervention in all of the three modalities. These results are presented in the Figures 2-4.

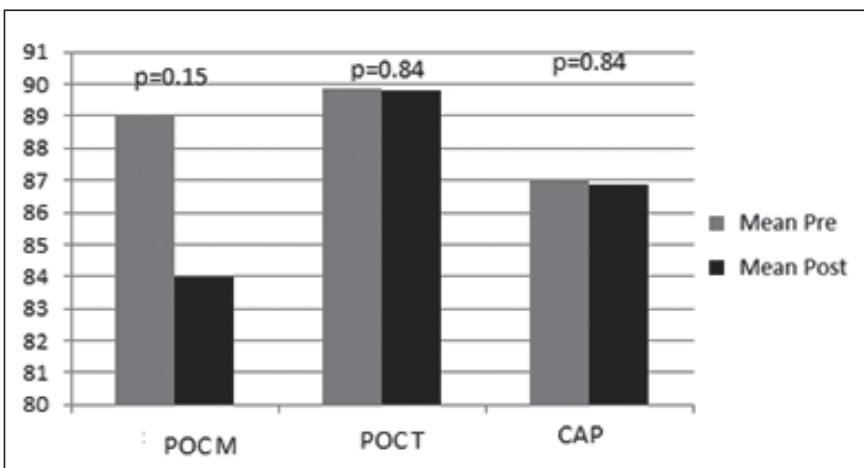

Fig. 2. Mean value of the symmetry (\%) of the masseter muscle (POC M) and anterior temporalis muscle (POC T) and CAP, pre and post intervention (Placebo $-n=16$ )

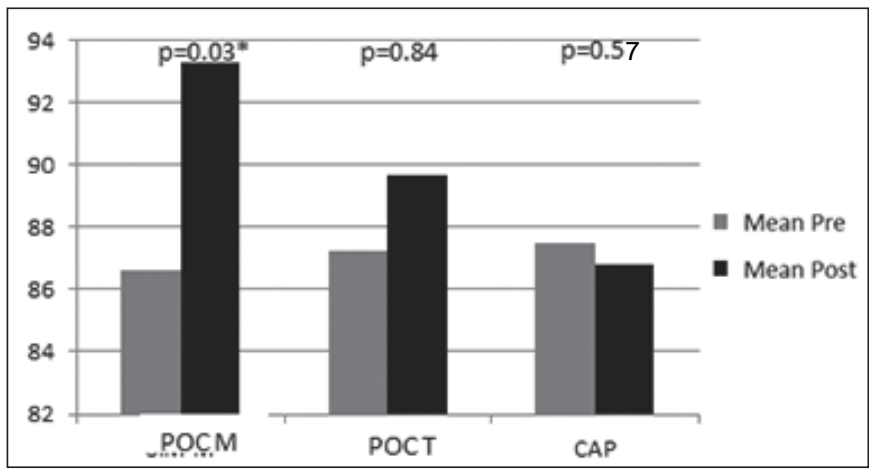

Fig. 3. Mean value of the symmetry (\%) of the masseter muscle (POC M) and anterior temporalis muscle (POC T) and CAP, pre and post intervention (Ultrasound- $n=16$ )

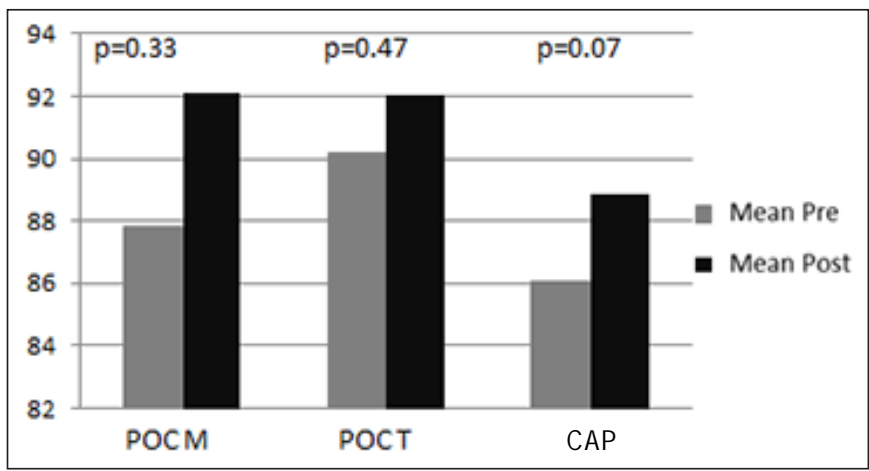

Fig. 4. Mean value of the symmetry (\%) of the masseter muscle (POC M) and anterior temporalis muscle (POC T) and CAP, pre and post intervention (Ultrasound with stretching - $n=16$ ).

\section{Discussion}

Studies regarding the specific and immediate effect of the ultrasound on the masticatory muscles activity were not found in the literature. Nevertheless, laser ${ }^{22}$ and $\mathrm{TENS}^{23}$ (transcutaneous electrical nerve stimulation) application as single interventions have been investigated, in addition to the immediate effect of the stabilization splint ${ }^{13,24}$.

A study evaluating the immediate effect of TENS on the EMG activity at rest in women with artrogenic $\mathrm{TMD}^{23}$ verified decrease in the sternocleidomastoid, digastric, masseter and anterior temporalis muscles activity after this intervention. Diversely, the present study evaluated the EMG activity during the maximum intercuspation after another modality of electrotherapy (ultrasound with stretching), by normalized EMG data, which hinders the comparison between the studies.

In the present study, the temporal anterior muscle presented higher values of the normalized EMG potentials than the masseter muscles during the maximum intercuspation, before and after the intervention. This result was also found by Ferrario et al. $^{25}(2000)$, but in healthy 
individuals with normal occlusion. The values of the EMG activity found in patients with TMD in the present study, during maximum intercuspation, were lower than the ones found in healthy individuals by Ferrario et al. ${ }^{25}$ (2000), suggesting a light level of dysfunction in these patients.

Mean POC values of $87.01 \%$ and $88.60 \%$ were observed by Tartaglia et al. ${ }^{16}$ (2011) in a EMG evaluation of masseter and anterior temporalis muscles, respectively, in women without TMD. Women with TMD presented lower values of symmetry, $85.85 \%$ and $84 \%$ for the masseter and anterior temporalis muscles, respectively. The symmetry values pre intervention of the present study resemble the control group results of the Tartaglia et al. study ${ }^{16}$.

The normalization of the EMG signal is recommended when comparing different subjects, days, muscles or studies ${ }^{19}$ and it consists in converting the data acquired in a given activity in percent values of a reference activity. However, not always the differences observed in RMS are confirmed after normalization ${ }^{26}$.

Botelho et al. ${ }^{13}$ (2010) found a significant increase in the masseter muscle symmetry during maximum intercuspation in 15 subjects with TMD after the use of a resilient splint. In this study, the POC values may be considered within normality compared to the values of healthy participants in the control group of the abovementioned study. Even so, a significant increase may be observed in the symmetry values of the masseter muscle after intervention with ultrasound combined to stretching.

Another study ${ }^{24}$ also verified, immediately after the use of stabilization splint, a significant increase in the symmetry of the masseter muscle, different from the temporalis muscle, which did not change after treatment.

The high APC values, possibly within the normality, previously to the intervention may explain the absence of statistically significant difference between pre and post intervention observed in this study, in all three modalities. Similar values were observed by Ferrario et al. ${ }^{18}$ (2006) in healthy subjects regarding the symmetry in the APC of masseter and anterior temporalis muscles. Based on this, it is believed that the participants of this study, apart from being diagnosed as TMD patients by RDC/TDM instruments, presented a slight degree of the dysfunction. The mentioned instruments do not allow determination of TMD severity ${ }^{27}$.

Before the interventions, the participants presented some EMG potential values within the normality patterns, probably due to adaptations. Acording to Türp et al. ${ }^{28}$ (2007), 75 95\% of acute TMD patients present significant improvements due to adaptive processes at the biological and psychological levels. This result also agrees with Manfredini et al. ${ }^{29}$ findings who observed spontaneous remission in most of TMD cases, regardless of the TMD diagnostic group, between the 2nd to 3rd year after the diagnosis. Therefore, as the patients of this study presented TMD for more than three months and did not carry out any physical therapeutic intervention in this period, it is possible that spontaneous improvements may have occurred. Besides, the myofascial TMD is categorized by episodes of symptoms exacerbation and remission ${ }^{30}$. These periods with symptoms remission may explain the EMG results found in this study, similar to the healthy subjects.

The present study presented some limitations, such as the sample size and the absence of TMD severity classification of the research subjects. It is believed that studies with more severely affected patients may demonstrate some evidence of immediate response after the intervention. Occlusal evaluation must also be included, since it may influence the EMG activity. In further studies, it is advisable to overcome these limitations.

The evaluation of craniocervical posture and the treatment of cervical pathology have been suggested for TMD patients $^{20}$. This study's findings, elicit the need of researches that investigate the duration, frequency and combination of therapeutic modalities, including the cervical vertebrae and muscles, in order to obtain positive and more definitive outcomes in the TMD treatment.

The results of this study showed that the ultrasound and ultrasound with masticatory muscle stretching did not modify the EMG potentials of the evaluated muscles with a single application, in women with myogenic and mixed TMD. However, the symmetry between the left and right masseter muscles increased immediately after the ultrasound with stretching.

\section{Acknowledgements}

We would acknowledge the CAPES for the support of this study.

\section{References}

1. Amaral AP, Politti F, Hage YE, Arruda EEC, Amorin CF, Biasotto-Gonzalez DA. Immediate effect of nonspecific mandibular mobilization on postural control in subjects with temporomandibular disorder: a single-blind randomized controlled clinical trial. Braz J Phys Ther. 2013; 17: 121-7.

2. La Touche R, Fernández-de-las-Peñas C, Fernández Carnero J, Escalante $\mathrm{K}$, Angulo-Díaz-Parreño S, Paris-Alemany A, et al. The effects of manual therapy and exercise directed at the cervical spine on pain and pressure pain sensitivity in patients with myofascial temporomandibular disorders. J Oral Rehabil. 2009; 36: 644-52

3. Carrara SV, Conti PCR, Barbosa JS. Statement of the 1st Consensus on Temporomandibular Disorders and Orofacial Pain. Dental Press J Orthod. 2010; 15: 114-20.

4. Cuccia AM, Caradonna C, Annunziata V, Caradonna D. Osteopathic manual therapy versus conventional conservative therapy in the treatment of temporomandibular disorders: a randomized controlled trial. J Bodyw Mov Ther. 2010; 14: 179-84.

5. Tosato J, Biasotto-Gonzalez DA, Caria PHF. Effect of massage therapy and of transcutaneous eletrical nerve stimulation on pain and electrotromyographic activity in patients with temporomandibular dysfunction. Fisioter Pesq. 2007; 14: 21-6.

6. Watson T. Ultrasound in contemporary physiotherapy practice. Ultrasonics. 2008; 48: 321-9.

7. Behm DG, Peach A, Madiggan M, Aboodarda SJ, Disanto MC, Button DC. Et al. Massage and streching reduce spinal reflex excitability without affecting twitch contractile properties. J Electromyogr Kinesiol. 2013; 23 : 1215-21.

8. Pinho JC, Caldas FM, Mora MJ, Santana-Penín U. Electromyographic activity in patients with temporomandibular disorders. J Oral Rehabil. 2000; 27 : 985-90 
9. Botelho AL, Brochini APZ, Martins MM, Melchior MO, Sllva AMBR, Silva MAMR. An electromyographic assessment of masticatory muscles asymmetry in normal occlusion subjects. Rev Odontol Univ São Paulo. 2008; 13: 7-12.

10. Nikolakis P, Erdogmus B, Kopf A, Djaber-Ansari A, Piehslinger E, FialkaMoser V. Exercise therapy for craniomandibular disorders. Arch Phys Med Rehabil. 2000; 81: 1137-42.

11. Furto ES, Cleland JA, Whitman JM, Olson KA. Manual physical therapy interventions and exercise for patients with temporomandibular disorders. Cranio. 2006; 24: 283-91.

12. Felício CM, Melchior MO, Ferreira CLP, Rodrigues DSMAM. Otologic symptoms of temporomandibular disorder and effect of orofacial myofunctional therapy. Cranio. 2008; 26: 118-25.

13. Botelho AL, Silva BC, Gentil FHU, Sforza C, Silva MAMR. Immediate effect of the resilient splint evaluated using surface electromyography in patients with TMD. Cranio. 2010; 28: 265-73.

14. Petrie A, Sabin C. Medical Statistics. São Paulo: Roca; 2007.

15. Turner JA, Mancl L, Huggins KH, Sherman JJ, Lentz G, LeResche L. Targeting temporomandibular disorder pain treatment to hormonal fluctuations: A randomized clinical trial. Pain. 2011; 152: 2074-84.

16. Tartaglia GM, Lodetti G, Paiva G, De Felício CM, Sforza C. Surface electromyographic assessment of patients with long lasting temporomandibular joint disorder pain. J Electromyogr Kinesiol. 2011; 21: 659-64.

17. Biasotto-Gonzalez DA, Bérzin F, Costa JM, Gonzalez TO. Electromyographic study of stomatognathic system muscles during chewing of different materials. Electromyogr Clin Neurophysiol. 2010; 50: 121-7.

18. Ferrario VF, Tartaglia GM, GallettaA, Grassi GP. The influence of occlusion on jaw and neck muscle activity: a surface EMG study in healthy young adults. J Oral Rehabil. 2006; 33: 341-8.

19. Ferrario VF, Tartaglia GM, Luraghi FE, Sforza C. The use of surface electromyography as a tool in differentiating temporomandibular disorders from neck disorders. Man Ther. 2007; 12: 372-9.

20. Ries LGK, Alves MC, Bérzin F. Asymmetric activation of temporalis, masseter and sternocleidomastoid muscles in temporomandibular disorder patients. Cranio. 2008; 26: 59-64.

21. Pertes AP, Gross. SG. Clinical treatment of temporomandibular disorders and orofacial pain. São Paulo: Quintessense; 2005.

22. Sattayud S, Bralley P. A study of the influence of low intensity laser therapy on painful temporomandibular disorders patients. Laser Therapy. 2012; 21: 183-92.

23. Monaco A, Sgolastra F, Pietropaoli D, Giannoni M, Cataneo R. Comparison between sensory and motor transcutaneous electrical nervous stimulation on electromyographic and kinesiographic activity of patients with temporomandibular disorder: a controlled clinical trial. BMC Musculoskelet Disord. 2013; 14: 2-8.

24. Ferrario VF, Sforza C, Tartaglia GM, Dellavia C. Immediate effect of a stabilization splint on masticatory muscle activity in temporomandibular disorder patients. J Oral Rehabil. 2002; 29: 810-5.

25. Ferrario V, Sforza C, Colombo A, Ciusa V. An electromyographic investigation of masticatory muscles symmetry in normo-occlusion subjects. J Oral Rehabil. 2000; 27: 33-40.

26. Kroll CD, Bérzin F, Alves M. Clinical evaluation of activity of masticatory muscles during usual mastication - a study on the normalization of EMG data. Rev Odontol UNESP. 2010; 39: 157-62.

27. Milanesi J, Weber P, Pasinato F, Corrêa ECR. Severity of the temporomandibular disorder and its relationship with craniocervical cephalometric measures. Fisioter Mov. 2013; 26: 79-86.

28. Türp JC, Jokstad A, Motschall E, Schindler HJ, Windecker-Gétaz I, Ettlin DA. Is there a superiority of multimodal as opposed to simple therapy in patients with temporomandibular disorders? A qualitative systematic review of literature. Clin Oral Implants Res. 2007; 18: 138-50.

29. Manfredini D, Favero L. Gregorini G, Cocilovo F, Guarda-Nardini L. Natural course of temporomandibular disorders with low pain-related impairment: a 2-to-3 year follow up study. J Oral Rehabil. 2013; 40: 436-42.
30. La Touche R, París-Alemany A, Von Piekartz H, Manheimmer JS, Fernández-Carnero J, Rocabado M. The influence of cranio-cervical posture on maximal mouth opening and pressure pain threshold in patients with myofascial temporomandibular pain disorders. Clin J Pain. 2011; 27: 48-55. 